\title{
Response assessment of a new albedo neutron dosimeter
}

\author{
Silvia Barros \\ Department of Nuclear Engineering, Sejong University \\ 209 Neungdong-ro, Gwangjin-gu, Seoul, South Korea \\ silviabarros@sejong.ac.kr \\ Geehyun Kim* \\ Department of Nuclear Engineering, Sejong University \\ 209 Neungdong-ro, Gwangjin-gu, Seoul, South Korea \\ gkim01@sejong.ac.kr
}

Published 10 July 2018

\begin{abstract}
The use of thermoluminescent dosimeters (TLDs) by personnel who work in radiation-rich environments is required by law. However, many professionals prefer to use Electronic Personal Dosimeters (EPDs), which provide dose estimation in real time. This preference may lead to a generalized use of the EPD instead of using the required TLD, as the use of both types at once can be uncomfortable and impractical. In an effort to avoid this scenario, a gamma/neutron dosimeter composed of a TLD and an EPD is being developed. In this paper, the results obtained from the studies performed in order to develop the neutron albedo dosimeter to be incorporated in the TLD+EPD dosimeter are presented. Monte Carlo simulations using the state-of-the-art Monte Carlo N-Particle Transport (MCNPX) code were used to calculate the response of the albedo dosimeter. The thermal neutron detectors under consideration for use in the proposed dosimeter are the EJ-426 and the EJ-420. Both detectors employ a lithium compound enriched to $95 \%{ }^{6} \mathrm{Li}$ dispersed in a ZnS:(Ag) matrix. The tomographic phantom Korean Typical Man-2 was used in the simulations to calculate the fraction of radiation backscattered by a human body in different radiation field conditions. From these results, it was concluded that both dosimeters are fit to be used as albedo dosimeters.
\end{abstract}

Keywords: Albedo dosimeter; Monte Carlo simulations.

\section{Introduction}

The use of thermoluminescent dosimeters (TLDs) by personnel who work in radiationrich environments is required by law in order to monitor the radiation they are exposed to and protect them from potential accidents that may arise from overexposure to radiation. However, many professionals prefer to use Electronic Personal Dosimeters (EPDs), which provide dose estimation in real time. This preference may lead to a generalized use of the EPD instead of using the required TLD, as the use of both types can be

*Corresponding author.

This is an Open Access article published by World Scientific Publishing Company. It is distributed under the terms of the Creative Commons Attribution 4.0 (CC-BY) License. Further distribution of this work is permitted, provided the original work is properly cited. 
uncomfortable and impractical and can result in underestimation of the doses delivered to workers.

In this paper, we present the preliminary work of the development of the neutron detector to be included in the integrated dosimeter that encloses both the required TLD and an EPD. The final goal of this work is to develop a light and easily portable card-type dosimeter that integrates TLD and EPD technologies, measures both gamma and neutron radiation, provides real-time dose estimation, and can be used in a wide range of work environments.

\section{Methods}

\subsection{Design and configuration of the neutron dosimeter}

The critical physical-design requirement of the dosimeter is that it be as small as possible (an enclosure about $5 \mathrm{~cm}$ wide) and be able to detect fast neutrons, so it was decided to use an albedo dosimeter for the neutron detection. This type of integrated dosimeter consists of two thermal neutron detectors. Both detectors are enclosed in a case thick enough to stop thermal neutrons. One of the dosimeters is located in front of a direct window (i.e., facing outward) and the other is placed in front of an albedo window facing the wearer (Fig. 1). The dosimeter located in the direct window measures the direct-field thermal neutron contribution, while the dosimeter in the albedo window measures the contribution of the fast-field neutrons moderated and backscattered by the body of the wearer. This configuration allows the measurement of neutrons over a wide range of energies (the minimum required limit is $0.025 \mathrm{eV}$ ) while achieving an optimal angular dependence between zero and 60 degrees.

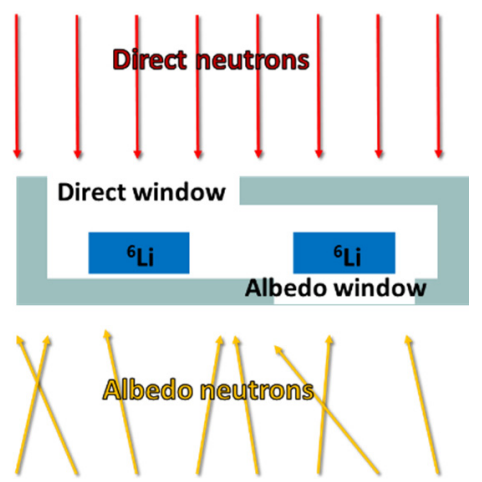

Fig. 1. Schematic diagram of a neutron albedo dosimeter.

\subsection{Thermal neutron detectors}

Two thermal neutron detectors were considered for use in this study: the EJ-426 and the EJ-420. ${ }^{1,2}$ The detection processes of both detectors depend on the nuclear reaction 
${ }^{6} \mathrm{Li}(\mathrm{n}, \alpha)$ to detect neutrons. The EJ-420 detector selected for this study was a $6.5-\mathrm{mm}$ thick disk $25 \mathrm{~mm}$ in diameter, while the EJ-426 detector was a $6 \mathrm{~mm} \times 6 \mathrm{~mm}$ square $0.5 \mathrm{~mm}$ thick.

\subsection{Monte Carlo simulations}

Monte Carlo simulations using the Monte Carlo N-Particle Transport (MCNPX) code ${ }^{3}$ were conducted to measure the responses of the neutron detectors, as well as their neutron energy, their angular dependence, and the dosimeter-to-body distance dependence. The scattered fraction of radiation being detected was also calculated during the simulations.

Figure 2 depicts the implemented models of the albedo dosimeters used in this study. In both cases, the thermal neutron detectors were enclosed in a $0.2 \mathrm{~cm}$ thick case made of borated polyethylene designed to stop direct and backscattered thermal neutrons.
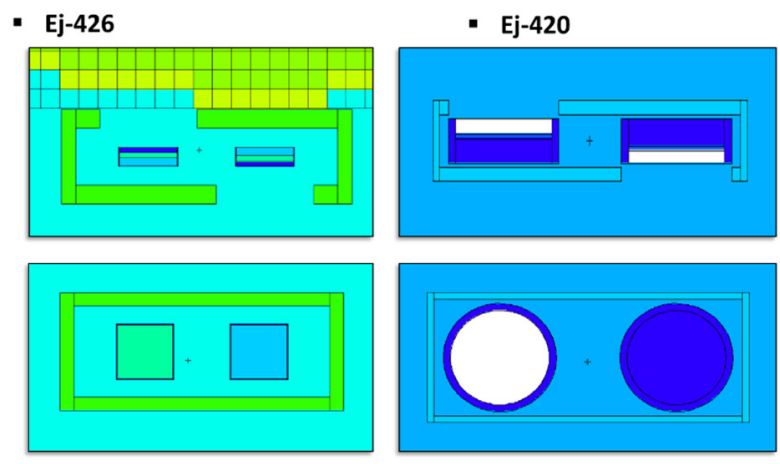

Fig. 2. Implemented models of albedo dosimeters used in this study: EJ-426 (left) and EJ-420 (right).

During the simulations, the albedo dosimeters were positioned at chest height (using the Korean Typical Man-2 tomographic phantom ${ }^{4}$ ) in order to simulate how a worker would wear a dosimeter. The $S(\alpha, \beta)$ thermal scattering treatment was used, so at thermal neutron energies (up to a few eV) thermal motion and chemical binding effects are taken into account. When this is not used, a free gas model is applied. The configuration of the neutron source used during simulation was planar and defined as a parallel beam with energies ranging from $0.01 \mathrm{eV}$ up to $10 \mathrm{MeV}$. Furthermore, the calibration of albedo dosimeters was performed with a $\mathrm{D}_{2} \mathrm{O}$ moderated ${ }^{252} \mathrm{Cf}$ field, ${ }^{5}$ in order to be used in proximity to particle accelerators, nuclear reactors, heavy shielding, and nuclear power stations. This configuration was also used in this study.

The MCNPX F4 card calculates the particle flux in a cell, and together with an fm card it can calculate the number of $(n, t)$ reactions in a material. Therefore, the responses of the detectors were obtained by counting the $(n, t)$ reactions in the ${ }^{6} \mathrm{Li}$ layer with the $\mathrm{F} 4$ and fm cards. In order to calculate the contribution from direct and albedo neutrons, the neutrons that were detected after interacting with the tomographic phantom were flagged. 


\section{Results}

\subsection{Scattered fraction}

Figures 3-4 and Tables 1-2 show the contribution from albedo and direct radiation for the models using the EJ-426 and EJ-420 neutron detectors. The direct contribution for the dosimeter made with the EJ-426 neutron detector is higher than $50 \%$ for energies below $1 \mathrm{eV}$ and is cancelled $(<5 \%)$ for energies above $1 \mathrm{keV}$. For the dosimeter using the EJ420 detector, the direct contribution is most relevant for energies up to $10 \mathrm{eV}$ and cancelled $(<5 \%)$ for energies above $10 \mathrm{keV}$.

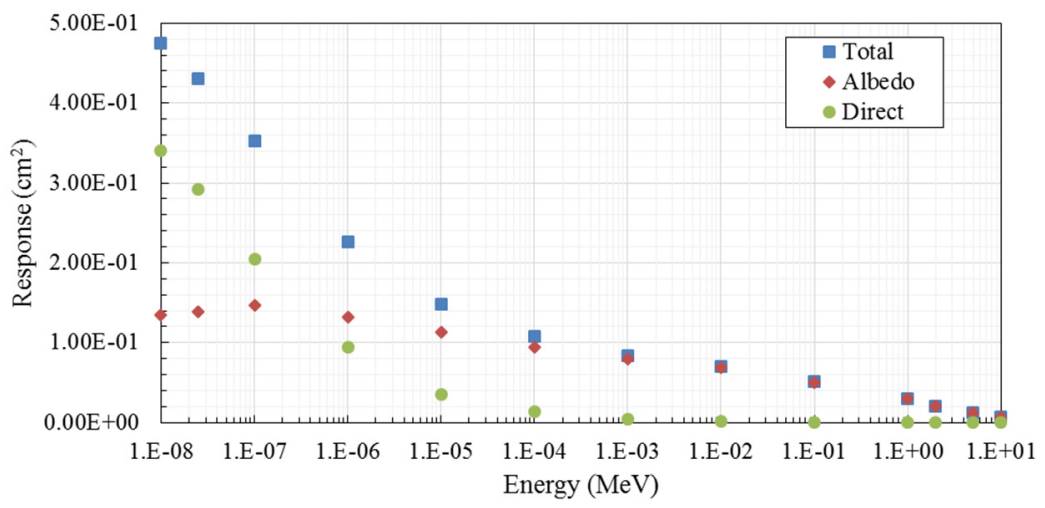

Fig. 3. Energy response of the albedo dosimeter implemented with the EJ-426 thermal neutron detector. The direct and albedo contributions are discriminated.

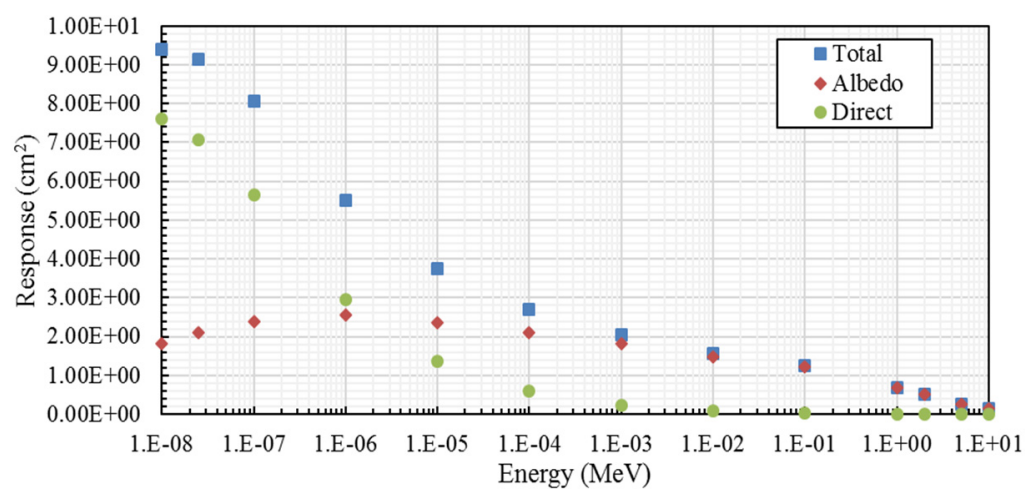

Fig. 4. Energy response of the albedo dosimeter implemented with the EJ-420 thermal neutron detector. The direct and albedo contributions are discriminated. 
Table 1. Scattered fraction of the albedo dosimeters implemented with EJ-426 and EJ-420 neutron detectors for energies between $1 \mathrm{E}-8$ and $1 \mathrm{E}-3 \mathrm{MeV}$.

\begin{tabular}{ccccccccc}
\hline & $\begin{array}{l}\text { Energy } \\
(\mathrm{MeV})\end{array}$ & $1.00 \mathrm{E}-08$ & $2.50 \mathrm{E}-08$ & $1.00 \mathrm{E}-07$ & $1.00 \mathrm{E}-06$ & $1.00 \mathrm{E}-05$ & $1.00 \mathrm{E}-04$ & $1.00 \mathrm{E}-03$ \\
\hline \multirow{2}{*}{$\mathrm{SF}$} & EJ-426 & $28 \%$ & $32 \%$ & $42 \%$ & $58 \%$ & $76 \%$ & $88 \%$ & $95 \%$ \\
& EJ-420 & $19 \%$ & $23 \%$ & $30 \%$ & $46 \%$ & $63 \%$ & $78 \%$ & $89 \%$ \\
\hline
\end{tabular}

Table 2. Scattered fraction of the albedo dosimeters implemented with EJ-426 and EJ-420 neutron detectors for energies between $1 \mathrm{E}-2$ and $1 \mathrm{E}+1 \mathrm{MeV}$.

\begin{tabular}{cccccccc}
\hline & $\begin{array}{c}\text { Energy } \\
(\mathrm{MeV})\end{array}$ & $1.00 \mathrm{E}-02$ & $1.00 \mathrm{E}-01$ & $1.00 \mathrm{E}+00$ & $2.00 \mathrm{E}+00$ & $5.00 \mathrm{E}+00$ & $1.00 \mathrm{E}+01$ \\
\hline \multirow{2}{*}{$\mathrm{SF}$} & EJ-426 & $98 \%$ & $99 \%$ & $99 \%$ & $99 \%$ & $99 \%$ & $100 \%$ \\
& EJ-420 & $95 \%$ & $98 \%$ & $99 \%$ & $99 \%$ & $99 \%$ & $99 \%$ \\
\hline
\end{tabular}

\subsection{Dosimeter-to-body distance dependence}

In order to study the dependence of the dosimeter's response upon its distance to the tomographic phantom, the detector was placed at distances of $0 \mathrm{~cm}, 1 \mathrm{~cm}, 2 \mathrm{~cm}$, and $3 \mathrm{~cm}$ from the phantom's chest. The results were obtained with a $\mathrm{D}_{2} \mathrm{O}+{ }^{252} \mathrm{Cf}$ neutron field (Table 3), since this is the recommended calibration field for the relevant application areas of this work (reactor and accelerators, nuclear power stations, heavy shielding and therapy particle reactors). ${ }^{5}$ The maximum deviation of the response was $18 \%$ for the dosimeter using the EJ-426 detector and 13\% for the dosimeter using the EJ-420 detector.

Table 3. Distance-to-body response of the EJ-426 and EJ-420 albedo dosimeters.

\begin{tabular}{cccccc}
\hline \multicolumn{5}{c}{ Distance to phantom $(\mathrm{cm})$} \\
\hline \multirow{4}{*}{ EJ-426 } & $0 \mathrm{~cm}$ & $1 \mathrm{~cm}$ & $2 \mathrm{~cm}$ & $3 \mathrm{~cm}$ \\
& & $9.03 \mathrm{E}-2$ & $7.88 \mathrm{E}-2$ & $7.44 \mathrm{E}-2$ & $7.46 \mathrm{E}-2$ \\
& Response $\left(\mathrm{cm}^{2}\right)$ & $\pm 5.60 \mathrm{E}-3$ & $\pm 4.85 \mathrm{E}-3$ & $\pm 4.72 \mathrm{E}-3$ & $\pm 2.45 \mathrm{E}-3$ \\
& Deviation from 0 cm & & $13 \%$ & $18 \%$ & $17 \%$ \\
& & 2.11 & 1.92 & 1.86 & 1.83 \\
EJ-420 & Response $\left(\mathrm{cm}^{2}\right)$ & $\pm 5.59 \mathrm{E}-2$ & $\pm 5.19 \mathrm{E}-2$ & $\pm 5.12 \mathrm{E}-2$ & $\pm 5.12 \mathrm{E}-2$ \\
& Deviation from 0 cm & & $9 \%$ & $12 \%$ & $13 \%$ \\
\hline
\end{tabular}

\subsection{Angular dependence}

In order to study the angular dependence of the dosimeter (Table 4), the angle of incidence of the $\mathrm{D}_{2} \mathrm{O}+{ }^{252} \mathrm{Cf}$ neutron field was varied between $0^{\circ}$ and $60^{\circ}$. For both dosimeters, the deviation of the response for $60^{\circ}$ was $46 \%$ in relation to $0^{\circ}$. The ISO 
21909 standard $^{6}$ stipulates that for an albedo dosimeter, the arithmetic mean of the response for $0^{\circ}, 15^{\circ}, 45^{\circ}$, and $60^{\circ}$ must not differ by more than $30 \%$ from the corresponding response at $0^{\circ}$. The deviation of the arithmetic mean is $19 \%$ for both dosimeters, so the angular dependence is within the requirements.

Table 4. Angular dependence of the EJ-426 and EJ-420 albedo dosimeters.

\begin{tabular}{|c|c|c|c|c|c|}
\hline & \multicolumn{5}{|c|}{ Angle of incidence } \\
\hline & & $0^{\circ}$ & $15^{\circ}$ & $45^{\circ}$ & $60^{\circ}$ \\
\hline \multirow{3}{*}{ EJ-426 } & Resnonse $\left(\mathrm{cm}^{2}\right)$ & $7.90 \mathrm{E}-2$ & $7.35 \mathrm{E}-2$ & $5.93 \mathrm{E}-2$ & $4.28 \mathrm{E}-2$ \\
\hline & Response $\left(\mathrm{cm}^{2}\right)$ & $\pm 2.05 \mathrm{E}-3$ & $\pm 2.84 \mathrm{E}-3$ & $\pm 2.45 \mathrm{E}-3$ & $\pm 3.14 \mathrm{E}-3$ \\
\hline & Deviation from $0^{\circ}$ & & $7 \%$ & $25 \%$ & $72 \%$ \\
\hline \multirow{3}{*}{ EJ-420 } & Pernons $\left(\mathrm{sm}^{2}\right.$ & 1.92 & 1.84 & 1.40 & 1.04 \\
\hline & Response $\left(\mathrm{cm}^{2}\right)$ & $\pm 5.19 \mathrm{E}-2$ & $\pm 5.14 \mathrm{E}-2$ & $\pm 4.34 \mathrm{E}-2$ & $\pm 3.79 \mathrm{E}-2$ \\
\hline & Deviation from $0^{\circ}$ & & $4 \%$ & $27 \%$ & $46 \%$ \\
\hline
\end{tabular}

\section{Conclusions}

In this work, two thermal neutron detectors, the EJ-420 and EJ-426, were used to construct the neutron dosimeter developed for this study. The angular dependence of both dosimeters is shown to meet the stated technical requirements. Concerning the dosimeterto-body distance dependence, the EJ-420 neutron detector displayed a smaller dosimeterto-body distance dependence $(<13 \%)$ than that of the dosimeter using the EJ-426 detector $(<18 \%)$. Both detectors were shown to be fit for use in albedo dosimeters. However, the use of the EJ-420 detector would result in a higher response dosimeter that requires a larger enclosure (at least $7 \mathrm{~cm}$ wide) that would exceed the desired $5 \mathrm{~cm}$ width.

\section{Acknowledgements}

This research has been supported by the National Research Foundation of South Korea (NRF-2016M2A2A4A04913625).

\section{References}

1. Thermal Neutron Detector EJ-426 (datasheet): http://www.eljentechnology.com/images/products/data_sheets/EJ-426.pdf , Eljen Technology (2016).

2. Thermal Neutron Detector EJ-420 (datasheet): http://www.eljentechnology.com/images/products/data_sheets/EJ-420.pdf, Eljen Technology (2016).

3. D. B. Pelowitz, ed., MCNPX User's Manual, Version 2.7.0, Los Alamos National Laboratory Technical Report LA-CP-11-0438 (April 2011).

4. C. Lee, C. Lee, S. H. Park and J. K. Lee, Med. Phys. 33(2), 380 (2006).

5. E. Piesch and B. Burgkhardt, Radiat. Prot. Dosim. 23, 117 (1988).

6. Radiation Protection e Passive Personal Neutron Dosemeters e Performance and Test Requirements, Standard ISO 21909 (ISO, Geneva, 2005). 